\title{
Erratum to: Low versus high radioiodine activity to ablate the thyroid after thyroidectomy for cancer: a meta-analysis of randomized controlled trials
}

Peizhun Du • Xuelong Jiao • Yanbing Zhou •

Yu Li $\cdot$ Shan Kang $\cdot$ Dongfeng Zhang •

Jizhun Zhang $\cdot$ Liang Lv $\cdot$ Rajan Patel

Published online: 12 September 2014

(C) Springer Science+Business Media New York 2014

\section{Erratum to: Endocrine}

DOI 10.1007/s12020-014-0333-8

Unfortunately, Rajan Patel was not included as one of the authors in the original publication of the article. The correct and complete order of authors and Rajan Patel's affiliation are provided in this erratum.

The online version of the original article can be found under doi:10.1007/s12020-014-0333-8.

P. Du · X. Jiao · Y. Zhou $(\bowtie) \cdot$ Y. Li · R. Patel

Department of General Surgery, Affiliated Hospital of Qingdao University, 16 Jiangsu Road, Qingdao 266003, China

e-mail: zhouyanbing999@126.com

\section{S. Kang $\cdot$ D. Zhang}

Department of Epidemiology and Health Statistics, The Medical College of Qingdao University, Qingdao, China

J. Zhang · L. Lv

Department of Gastrointestinal Surgery, Laboratory of Surgical Oncology, Peking University People's Hospital, Beijing, China 